\title{
EDITORIAL
}

\section{Las guías de práctica clínica y el ejercicio médico}

$\mathrm{H}$ ace ya casi 20 años, durante mi especialización en Epidemiología General, uno de los aspectos más importantes sobre el cual se hacía mucho énfasis era la búsqueda de respuestas a una pregunta de la práctica clínica. Era importante saber hacer la pregunta, era importante saber organizar en las bases de datos, las palabras claves sobre las cuales hacer la búsqueda, una vez hecha ésta, los criterios con los cuales iba a seleccionar los artículos más adecuados para contestar mi pregunta y una cosa importantísima finalmente era, después de todo este proceso, saber si la población de los artículos más útiles era representativa y los resultados eran aplicables a nuestra población en general y a mi paciente en particular. Es tal la avalancha de información publicada actualmente en revistas médicas que sería imposible leer si quiera el 1\% de lo publicado en el mundo en el último año sobre un tema en particular, por lo que surgen los estudios de metanálisis, las revisiones de literatura y publicaciones que tratan de resumir de la mejor forma metodológica lo último en avances en un campo en particular. Si a esto le agregamos la necesidad que tienen los servicios de salud de prestar la atención lo más eficientemente posible, en términos financieros, para la mayor cantidad de población posible, queda listo el terreno para la aparición de lo que será el ascenso y caída del ejercicio de la Medicina, las guías de práctica clínica.

¿Por qué el ascenso? Gracias a la aparición de las guías de práctica clínica, el médico puede rápidamente aplicar la mejor calidad de atención a su paciente, basándose en un documento que lo orienta según la mejor evidencia estadística disponible en ese momento. Hay una racionalización de los gastos en salud al orientar la solicitud de los exámenes más adecuados y pertinentes para una patología y facilitar la enseñanza de la medicina, ya que al enseñar y/o aprender la guía de manejo, el estudiante o el profesional son capaces de manejar de una manera racional la patología objeto de la misma.

¿Por qué la caída? Porque la MBE (Medicina Basada en la Evidencia), filosofía y eje de todas las guías, deja a un lado el paciente y convierte el ejercicio de la medicina en una profesión técnica que debe ajustarse a protocolos y guías de manejo, útiles en términos estadísticos y poblacionales como una receta que se debe seguir, dejando a un lado a la ciencia y arte del ejercicio de la profesión en donde la MBE permite que el profesional tenga el suficiente criterio para decidir si una terapia o un abordaje diagnóstico puede ser el más eficaz y eficiente en su paciente en particular, así en la evidencia estadística no sea la mejor vía o abordaje en términos poblacionales y estadísticos. Del mismo modo, como lo comentaba al inicio de este documento, un pilar fundamental de la MBE es que la aplicación de la evidencia sea posible en términos poblacionales y aquí es donde veo que cometemos un gran error. No puedo desconocer la calidad e importancia del desarrollo de guías de manejo de patologías frecuentes, pero lo que no entiendo es por qué aplicamos una guía extranjera a nuestra población. Nosotros como endocrinólogos y creo que es una situación que se repite en todas las especialidades, aplicamos las guías de manejo de la Asociación Americana de Endocrinología Clínica, o las guías de la Endocrine Society o las guías de la Asociación Europea de Endocrinología para la mayoría de las patologías en donde hay un documento de este tipo. Pero, salvo algunas excepciones de médicos endocrinólogos con entrenamiento en epidemiología clínica, nunca nos detenemos a analizar la población sobre la que se hicieron los estudios soporte del desarrollo de dichas guías y, sin embargo, las aplicamos en nuestros pacientes; es más, en ocasiones criticamos conductas seguidas por un profesional, basándonos en que la guía de manejo dice otra cosa.

Esto me lleva a una última reflexión. ¿Cuál es el material soporte para el desarrollo de guías? Lo que publicamos. Y, ¿qué publicamos? Lo que nos reciban para publicación en las revistas internacionales y nos permitan publicar los comités de investigaciones en donde habrá una persona que diga que ese estudio no tiene el suficiente soporte estadístico para ser publicado, quiere decir que la gran masa de literatura médica tiene un sesgo, el sesgo de publicación.

Parte de mi motivación para promover la aparición de la revista de la Asociación es que tengamos un sitio en dónde publiquemos el material suficiente que nos permita desarrollar nuestras propias guías, basándonos en nuestra literatura y nuestra población y sabiendo que con la filosofía de que los estudios originales son la base para que una revista sea reconocida internacionalmente como de calidad, este no será un objetivo a corto plazo. Tendremos que sacrificar en cierta medida dicho objetivo, publicando estudios de serie de casos, experiencias, casos clínicos y todo el material, no significativo estadísticamente, que permita tener el suficiente bagaje documental que nos permita tener nuestros propios protocolos y guías. Así que los invito a todos a publicar.

Cordialmente,

\section{William Rojas García, MD}

